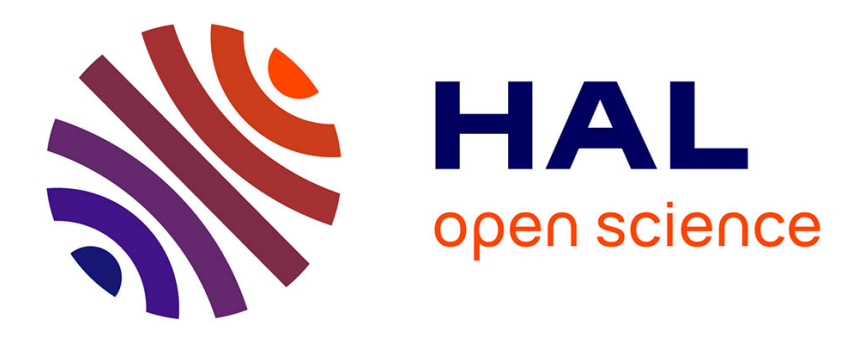

\title{
Numerical model of static hysteresis taking into account temperature
}

\author{
Fabien Sixdenier, Riccardo Scorretti
}

\section{To cite this version:}

Fabien Sixdenier, Riccardo Scorretti. Numerical model of static hysteresis taking into account temperature. International Journal of Numerical Modelling: Electronic Networks, Devices and Fields, 2017, 10.1002/jnm.2221 . hal-01497369

\section{HAL Id: hal-01497369 \\ https://hal.science/hal-01497369}

Submitted on 28 Mar 2017

HAL is a multi-disciplinary open access archive for the deposit and dissemination of scientific research documents, whether they are published or not. The documents may come from teaching and research institutions in France or abroad, or from public or private research centers.
L'archive ouverte pluridisciplinaire HAL, est destinée au dépôt et à la diffusion de documents scientifiques de niveau recherche, publiés ou non, émanant des établissements d'enseignement et de recherche français ou étrangers, des laboratoires publics ou privés. 


\title{
Numerical model of static hysteresis taking into account temperature
}

\author{
Fabien Sixdenier \\ email fabien.sixdenier@univ-lyon1.fr \\ Riccardo Scorretti \\ email riccardo.scorretti@univ-lyon1.fr \\ Univ Lyon, UCB Lyon 1, CNRS, AMPERE, F-69100, \\ VILLEURBANNE, France \\ March 28, 2017
}

\begin{abstract}
Some soft magnetic materials are strongly dependent of the temperature, because of their low Curie temperature. In order to predict their behaviour in electrical devices, hysteresis models able to take into account the temperature are needed. The Vector Play static hysteresis model is an interesting hysteresis model that can be modified in order to take into account the thermal behaviour. In this paper, the temperature is taken into account in this last model through its parameters. The variations of some parameters with temperature are mainly issued from numerical interpolation and specific assumptions. Simulation results are compared to measurements and discussed.
\end{abstract}

\section{Introduction}

The well known Jiles-Atherthon 1 and Preisach 2 hysteresis models were widely used during the last decades to predict hysteresis loops or magnetic losses in many cases. Despite the huge number of users of these models, very few authors tried to take into account the temperature. For example [3] used analytical empirical laws of variation of the Jiles-Atherthon model parameters versus temperature to predict hysteresis loops. In 4, authors compared several approaches (analytical 3, numerical, and mixed) to identify each parameter variations. Concerning Preisach models, the authors of [5] used a double well potential distribution. Both static and dynamic interactions for an ultra fine particulate system were taken into account in order to simulate various magnetization processes, such as: field-cooled, zero field-cooled, thermo-remanent magnetization, magnetic relaxation in applied field, and the major hysteresis loop as a function of temperature. The authors of [6] assumed that the Preisach distribution function was a factorized product of a Gaussian coercive field distribution, and a Gaussian interaction field distribution both with some dispersion coefficients. Those last two approaches are rather complicated to implement in engineering softwares such as finite element or circuit-type (SPICE) ones. 
The vector play models (also called energy-based vector hysteresis model) are phenomenological models of hysteresis first introduced by Bergqvist [7] which have many desirable properties. In particular, it is intrinsically vectorial, and provides an accurate energy balance [8. It has been shown in 9 ] that the model accuracy was strongly improved by increasing the number of cells. However a relatively small number of internal variables was actually necessary to obtain an accurate representation of the complex response of the material. Therefore, a trade-off between accuracy and number of cells is always possible with this model. This is not the case for the Jiles Atherthon model for example.

The VPM has been tested and implemented in Finite Elements by many authors [9, 8, 10, and recently been improved in the case of rotating fields [1]. So as to be useful in practical engineering applications, models must consider the effect of temperature. The same authors also proposed a method 12 where they considered linear variations of saturation magnetization and coercive field to take into account the temperature for permanent magnets. In a previous paper [13], variations of some parameters of the VPM were identified thanks to different papers in the literature [3, 14, 15]. This last approach gave mixed results. First, the purely magnetic behaviour gave not so good results for very low applied fields (not enough accurate description in this particular region). Second, the VPM showed no difficulties to adapt itself to the temperature dependence, by allowing some parameters to change their values. The thermal behaviour was qualitatively well retrieved, loops expanded or shrank themselves in function of temperature showing the ability of the build model to qualitatively retrieve the Hopkinson effect.

In order to improve the accuracy of the simulations of the VPM, we present in this work recent developments on the dependence of VPM on temperature. In our approach, no analytical (empirical) laws are used in order to predict the thermal behaviour. Secondarily, a novel approach for identifying VPM's parameters is proposed.

The paper is organized as follows: the implemented VPM and the main assumptions made to take into account the temperature are presented (section 2). The identification of VPM's parameters is analysed in section 3 . In section 4, the results of the identification algorithm are presented on a virtual and a real material. Discussion of obtained results and perspectives conclude the paper (section 5).

\section{The vector play model}

\subsection{Purely magnetic behaviour}

Hysteresis in magnetic materials can be seen as defects at microscopical scale, which pin Bloch walls and prevent them from moving under the effect of an external magnetic field. When the magnetic energy becomes higher than the energy barrier due to this pinning force, Bloch walls suddenly rearrange and the energy corresponding to the jump is dissipated [7].

Basing on an analogy between pinning force and mechanical friction force, Henrotte et al. proposed a phenomenological (macroscopic) model which is consistent with laws of thermodynamics [8]. In this model, the applied magnetic 

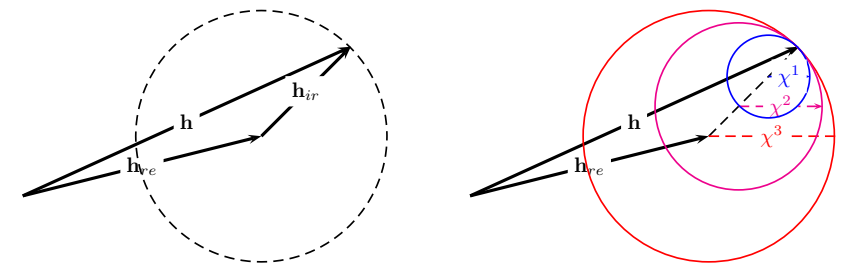

Figure 1: Vector diagram of $\mathbf{h}, \mathbf{h}_{r e}$ and $\mathbf{h}_{i}$ for a single cell (left), and for $N=3$ cells (right).

field $\mathbf{h}$ is decomposed in a reversible $\mathbf{h}_{r e}$ and irreversible $\mathbf{h}_{i r}$ part:

$$
\mathbf{h}=\mathbf{h}_{r e}+\mathbf{h}_{i r}
$$

The magnetic field $\mathbf{h}$ is the input of the model. The reversible magnetic field $\mathbf{h}_{r e}$ is computed as the weighted sum of contributions $\mathbf{h}_{r e}^{k}$ of a number $N$ of "cells" (in practice $\mathbf{h}_{i r}$ is never computed explicitly). Each cell is analogous to a mechanical system composed of a spring and a friction slider:

$$
\mathbf{h}_{r e}^{k}=\sum_{k=1}^{N} \omega_{k} \mathbf{h}_{r e}^{k}
$$

In this sum, $\omega_{k} \geq 0$ are the weights associated to cells, and must verify the condition: $\sum_{k=1}^{N} \omega_{k}=1$. The terms $\mathbf{h}_{r e}^{k}$ are the internal state of each cell. They are upgraded as a function of the applied magnetic field $\mathbf{h}$ and of their current value $\mathbf{h}_{r e 0}^{k}$ according to:

$$
\mathbf{h}_{r e}^{k}= \begin{cases}\mathbf{h}_{r e 0}^{k} & \text { if }\left\|\mathbf{h}-\mathbf{h}_{r e 0}^{k}\right\|<\chi^{k} \\ \mathbf{h}-\chi^{k} \cdot \frac{\mathbf{h}-\mathbf{h}_{r e 0}^{k}}{\left\|\mathbf{h}-\mathbf{h}_{r e 0}^{e}\right\|} & \text { otherwise }\end{cases}
$$

where $\chi^{k}$ is a parameter linked to the coercivity [13. The relationship between $\mathbf{h}, \mathbf{h}_{r e}$ and $\mathbf{h}_{i}$ is depicted in figure 1 1 . The magnetization $\mathbf{M}$ is computed as a function of the reversible magnetic field as:

$$
\mathbf{M}=M_{a n}\left(\left\|\mathbf{h}_{r e}\right\|\right) \cdot \frac{\mathbf{h}_{r e}}{\left\|\mathbf{h}_{r e}\right\|}
$$

where $M_{a n}\left(\left\|\mid \mathbf{h}_{r e}\right\|\right)$ is a scalar anhysteretic magnetization function. Finally, the magnetic flux density is computed as:

$$
\mathbf{b}=\mu_{0}(\mathbf{M}+\mathbf{h})
$$

One of the advantages of this model is that it is intrinsically vectorial. Moreover, the number of cells is not fixed a priori, and can eventually be adjusted in such a way to fulfill accuracy requirements.

\subsection{Temperature dependent extension}

All equations and principles of the model seen before remain valid, only some parameters will have their value changed. As seen in the previous section, the VPM 
is mainly governed by the anhysteretic function $M_{a n}$ and by the $\chi^{k}$ values. For real materials, analytical anhysteretic functions (see [8] for possible analytical functions) always present more or less error on one or several regions (saturation, saturation knee, low field region) compared to the measured anhysteretic curve. Moreover, analytical functions also need an identification algorithm of physical (best case) or empirical (worst case) parameters. As the temperature will change, all anhysteretic function parameters will change as well. If some physical parameters variations with temperature are well described in the literature (example : saturation magnetization $M_{s}(T)$ ), some empirical parameters are not. For these reasons, we chose to use a specific interpolation table in order to evaluate $M_{a n}$ at a given temperature.

For anhysteretic magnetization $M_{a n}$, several major loops are measured at different temperatures that were performed on a ring sample wound with high temperature enameled wires (placed in an thermal enclosure) following the fluxmetric method with respect to the IEC 60404-4 standard [16. More precisely, the major loop is measured at several temperatures, and the anhysteretic magnetization $M_{a n}(h, T)$ is computed by considering the middle point of horizontal lines which connect ascending and descending branches of the (major) loop. The anhysteretic magnetization function $M_{a n}(h, T)$ for a generic temperature $T$ is obtained by interpolation. The figure 2 (left) shows the built $2 \mathrm{D}$ anhysteretic magnetization $M_{a n}$ versus the temperature $T$ and of the applied field $h$. These data are measured on the same material than the one presented in section 4.2 .
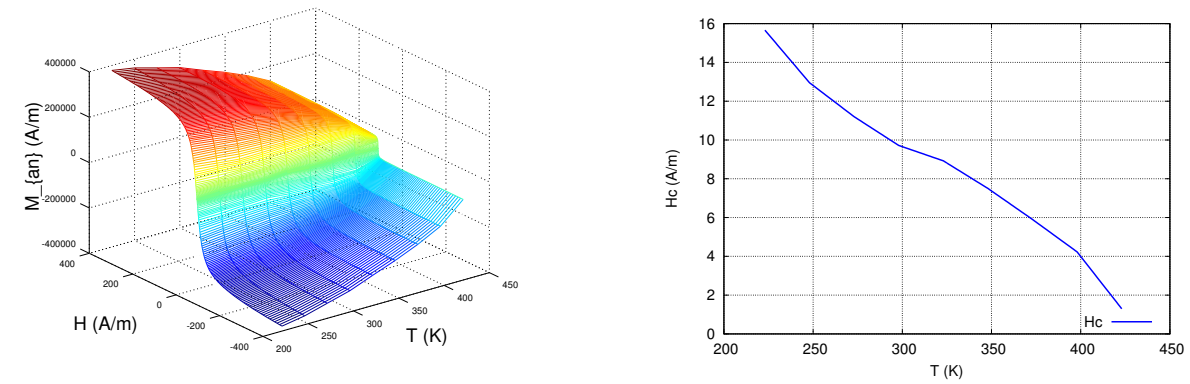

Figure 2: Left: Anhysteretic magnetization $M_{a n}$ versus the temperature $T$ and of the applied field $H$. Right: Coercive field $H_{c}$ versus temperature $T$. Measurements were made on a $M n Z n$ N30 ferrite material (see section 4).

As for parameters $\omega_{k}$, the same property $\left(\sum_{k=1}^{N} \omega_{k}=1\right)$ must hold at any temperature, we assume that in fact they don't depend on the temperature. However parameters $\chi^{k}$ are related to a local pinning force or a local coercive field; therefore it is assumed here that the variations of all $\chi_{k}$ with temperature are the same as the macroscopic coercive field $H_{c}$, which is already known with the measurements of major loops at different temperatures (figure 2 (right)). Now, let us suppose that all $\chi^{k}$ have been identified at room temperature (RT). All $\chi^{k}$ at any temperature $T$ within the range can be rescaled by applying (5).

$$
\chi^{k}(T)=\frac{H_{c}(T)}{H_{c}(R T)} \chi^{k}(R T)
$$

In order to verify the assumptions made here, the $\left(\omega_{k}, \chi^{k}\right)$ parameters have 
to be identified so as to that the VPM give accurate results for any kind of signals (low/high levels, single/multiple frequency(ies)).

\section{Parameters identification}

The VPM is fully characterized by the anhysteretic magnetization function $M_{a n}(h)$, and the $N$ couples of parameters $\left(\omega_{k}, \chi^{k}\right)$ which represent each of the $N$ cells which compose the model. The number $N$ of cells is a priori unknown.

The parameters $\omega_{k}$ and $\chi^{k}$ must verify the following constraints:

$$
\begin{array}{ll}
\omega_{k} \geq 0 \quad & ; \quad \sum_{k=1}^{N} \omega_{k}=1 \\
\chi^{1}=0 \quad ; \quad \chi^{k} \leq \chi^{k+1}
\end{array}
$$

In 8 , it is proposed to determine these parameters basing on the experimental curve $H_{c}\left(H_{p}\right)$, where $H_{c}$ is the coercitive field corresponding to a minor symmetric closed loop, for which the peak magnetic field is $H_{p}$. The same approach is followed in [10] and [13. However, this approach requires a specific set of measurements (i.e. symmetric closed loops at many peak levels), which have to be performed purposely. Conversely, it would be interesting to explore the possibility of being able to identify model parameters from a generic set of measurements. In this work we followed this second approach. We stress that the term "generic" does not mean that any dataset will suit, but rather that the excitation field $h(t)$ does not need to be a specific signal. This last point will be discussed later.

\subsection{Anhysteretic magnetization}

The anhysteretic magnetization function $M_{a n}(h, T)$ is obtained from experimental measurements taken at different temperatures (see previous section).

\subsection{Naive formulations for the identification problem}

Therefore the only parameters which have to be identified are $\omega_{k}$ and $\chi^{k}$ (at a given temperature $T$ ) with $k$ spanning from 1 and the (unknown) number of cells $N$. According to [13, these parameters have been fitted for a single temperature $T_{0}=298 \mathrm{~K}$; their variation with temperature is as described in section 2 . For sake of simplicity, let's define the vectors $Y=\left(\omega_{1} \ldots \omega_{N}\right)$ and $X=\left(\chi^{1} \ldots \chi^{N}\right)$. For a given number of cells $N$, and a given dataset of experimental measurement $b(h)$, one possibility would be to solve the constrained optimization problem:

$$
\left\{\begin{array}{l}
\left(\omega_{k}, \chi^{k}\right)=\arg \min _{Y, X}\left\|b(h)-b_{s}(h)\right\|_{2}^{2} \\
\omega_{k} \geq 0 ; \quad \sum_{k=1}^{N} \omega_{k}=1 \\
\chi^{1}=0 ; \quad \chi^{k} \leq \chi^{k+1}
\end{array}\right.
$$

where $b_{s}(h)$ is the magnetic flux density computed by the model, which clearly depends on the set of parameters $\left(\omega_{k}, \chi^{k}: k=1 \ldots N\right)$. In fact, the quadratic 
term $\left\|b(h)-b_{s}(h)\right\|^{2}$ could be replaced by any meaningfull cost function, for instance:

$$
\left\{\begin{array}{l}
\left(\omega_{k}, \chi^{k}\right)=\arg \min _{Y, X}\left\|H_{c}\left(H_{p}\right)-H_{c, s}\left(H_{p}\right)\right\|_{2}^{2} \\
\omega_{k} \geq 0 ; \quad \sum_{k=1}^{N} \omega_{k}=1 \\
\chi^{1}=0 ; \quad \chi^{k} \leq \chi^{k+1}
\end{array}\right.
$$

where $H_{c, s}\left(H_{p}\right)$ is the simulated coercive field, which can be easily evaluated 8 as:

$$
H_{c, s}\left(H_{p}\right)=\frac{\sum_{k=1}^{m\left(H_{p}\right)} \omega_{k} \chi^{k}}{\sum_{k=1}^{m\left(H_{p}\right)} \omega_{k}}
$$

where the index $m\left(H_{p}\right) \in[1 ; N]$ is the largest integer such that $\chi^{k} \leq H_{p} \forall k \leq$ $m\left(H_{p}\right)$.

\subsection{Improved formulation}

All these "naive" formulations are prone to several problems. One of them is that the optimization algorithm can stagnate in a local minimum. Before introducing better formulations, one can observe that a single cell $(\omega, \chi)$ can be replaced by two cells $\left(\omega_{1}, \chi^{1}\right)$ and $\left(\omega_{2}, \chi^{2}\right)$, provided that: $\omega=\omega_{1}+\omega_{2}$ and $\chi=\chi^{1}=\chi^{2}$. With more generality, a single cell $(\omega, \chi)$ can be replaced by a number $p$ of cells $\left(\omega_{i}, \chi\right)$ in the following way:

$$
(\omega, \chi) \Leftrightarrow\left(\omega_{1}, \chi\right) \ldots\left(\omega_{p}, \chi\right) \text { provided that: } \sum_{i=1}^{p} \omega_{i}=\omega
$$

Keeping this observation in mind, we can reformulate the problem (8) by considering a number $N$ of cells $\left(\omega, \chi^{k}\right)$, all of which share the same value of $\omega=\frac{1}{N}$ : in this way, the constraints (6) are fulfilled by construction:

$$
\left\{\begin{array}{l}
\left(\omega_{k}, \chi^{k}\right)=\arg \min _{X}\left\|b(h)-b_{s}(h)\right\|_{2}^{2} \\
\omega_{k} \equiv 1 / N \\
\chi^{1}=0 ; \quad \chi^{k} \leq \chi^{k+1}
\end{array}\right.
$$

The formulation (12) can be further simplified by taking as unknown the difference $\Delta \chi^{k}=\chi^{k+1}-\chi^{k}$ instead of $\chi^{k}$, which will be constrained to be nonnegative. In this way we obtain the following (nonlinear) non-negative least squares formulation:

$$
\left\{\begin{array}{c}
\Delta \chi^{k}=\arg \min _{\Delta X}\left\|b(h)-b_{s}(h)\right\|_{2}^{2} \\
\Delta \chi^{k} \geq 0
\end{array}\right.
$$

where $\Delta X=\left(\Delta \chi^{1} \ldots \Delta \chi N-1\right)$. Observe that formulation (8) has a number $2 N-1$ unknowns, whereas this formulation has only $N-1$ unknowns. However, due to the fact that in this formulation $\omega_{k}$ takes discrete values, in principle a finer discretization (i.e. a higher value of $N$ ) would be required so as to achieve comparable accuracy.

The most important improvement of formulation (13) with respect of (8) or (9) is that constraints are much more relaxed. Moreover, due to the equivalence property (11), the fact that in (8) $\omega_{k}$ are free parameters may introduce many 
local minima in the cost function. This is not the case with $(13)$ because $\omega_{k} \equiv$ $1 / N$ is imposed. Therefore the optimization problem should be easier to solve numerically.

The price to pay for this is that a higher number $N$ of cells has to be considered. This point is a huge drawback in view of Finite Element implementation. Therefore we devised an algorithm for reducing the number of cell. Namely, our strategy is to solve (13) with a high number of cells, and then simplify the obtained VPM by packing some cells together.

\subsection{Packing cells algorithm}

First of all, observe that the number of independent cells provided by formulation (13) corresponds to the number of non-zero values of $\Delta X$. In fact, two (or more) consecutive cells corresponding to $\Delta \chi^{k}=0$ can be packed together into a single cell by using the same argument (11) developed beforehand, that is:

$\Delta \chi^{k}=\ldots=\Delta \chi^{k+p-1}=0 \Leftrightarrow\left\{\begin{array}{c}\left(1 / N, \chi^{k}\right) \\ \left(1 / N, \chi^{k+1}\right) \\ \vdots \\ \left(1 / N, \chi^{k+p}\right)\end{array}=\left\{\begin{array}{c}\left(1 / N, \chi^{k}\right) \\ \left(1 / N, \chi^{k}\right) \\ \vdots \\ \left(1 / N, \chi^{k}\right)\end{array} \longrightarrow\left(\frac{p}{N}, \chi^{k}\right)\right.\right.$

The main idea of the algorithm is to consider the case of a VPM composed by an unknown, small number $N_{T}$ of independent cells, which is identified by solving the problem (13). In the ideal case (no measurement noise, exact arithmetic, etc.) the solution $\left(\Delta \chi^{k}\right)$ should be composed of exactly $N_{T}$ non-zero terms, whatever the discretization $N$. However in practice this is rarely the case. So, we are expecting that the obtained solution will be composed of at best $N_{T}$ non-zero terms, and other small but non-zero terms.

In order to recover the true solution, the idea is to search the smallest nonzero term, and to aggregate it with its bigger neighbour. Namely, assume that $\Delta \chi^{k}$ is the smallest non-zero term of $\Delta X$, and that $\Delta \chi^{k+1}>\Delta \chi^{k-1}$; then the algorithm will execute:

$$
\begin{array}{ll}
\Delta \chi^{k+1} & \leftarrow \Delta \chi^{k+1}+\Delta \chi^{k} \\
\Delta \chi^{k} & \leftarrow 0
\end{array}
$$

This simple iteration is repeated until it is possible to simplify the VPM, that is until two non-zero, not too small, adjacent terms can be found in the vector $\Delta X$. At each iteration a term of $\Delta X$ is set to 0 , so that this algorithm will necessarily terminate. The pseudocode of the algorithm is listed hereafter:

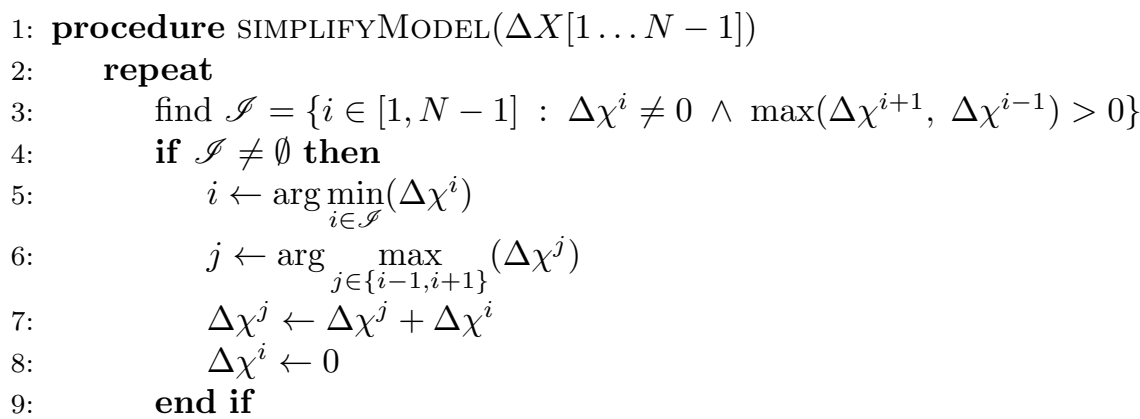




\section{0: $\quad$ until $\mathscr{I} \neq \emptyset$ \\ 11: end procedure}

\section{Results}

\subsection{Fake material}

Before using formulation (13) to identify a real material, we performed preliminary tests by using simulated measurements and validation datasets. The fake material we simulated has an anhysteretic magnetization described by a Langevin function:

$$
M_{a n}(h)=M_{s}\left(\operatorname{coth}\left(\frac{h}{h_{0}}\right)-\frac{h_{0}}{h}\right)
$$

with $M_{s}=4 \cdot 10^{5} \mathrm{~A} / \mathrm{m}$ and $h_{0}=7 \mathrm{~A} / \mathrm{m}$, and it is modeled by a VPM composed by only 3 cells:

$$
(0.1,0)-(0.3,5)-(0.6,15)
$$

The data generated by using this model have been fitted with formulation (13). So as to avoid inverse crime [17, in the generation of this synthetic data, we simulated a Gaussian white noise $\eta \sim \mathcal{N}\left(0,5^{2} \mathrm{mT}^{2}\right)$, and we introduced a bias of $1 \%$ in parameters $M_{s}$ and $h_{0}$. The minimization algorithm used is the interior point algorithm [18, as implemented in the function fmincon of MATLAB ${ }^{\circledR}$. All simulations have been repeated 100 times so as to evaluate confidence intervals by a Monte Carlo method [19]. The result of one of these is depicted in figure 3 . The values of $\left(\chi^{k}\right)$ obtained for $N=10$ are listed in table 1. The number of cells is the number of different values of $\chi^{k}$ : for instance, for $N=10$ formulation 13 provided the 4-cells simplified VPM (table 1, last column):

$$
(0.1,0)-(0.3,5.064)-(0.5,14.938)-(0.1,15.454)
$$

One observes that all formulations provide results which are quite close to the reference VPM (figure 3). VPMs with different numbers of cells can be compared by plotting $\chi^{k}$ as a function of the variable $\Omega_{k}=\sum_{i=1}^{k} \omega_{i}$. We performed simulations with $N$ spanning from 10 to 50: we found that the obtained results are quite stable (not shown).

The simplification algorithm is very effective in reducing the number of cells. For engineering purposes, one could mention that the computation of losses also provide accurate results up to $5 \%$ for all fitted models.

\subsection{Real material}

We identified different VPMs corresponding to an Manganese Zinc N30 ferrite material, which has been selected for its low value of $T_{c}<433 \mathrm{~K}$. The experimental measurements used for identification and validation are depicted in figure 4. For identification we used the formulation (13), with $N$ spanning from 10 to 50. Datasets used for identification and validation have been measured at $298 \mathrm{~K}$. The obtained values for $\left(\chi^{k}\right)$ are reported in table 2 for $N=10$.

One observes that the formulation (13) provided results in good agreement with experimental data (comparison of loops). Like in previous simulations, the 

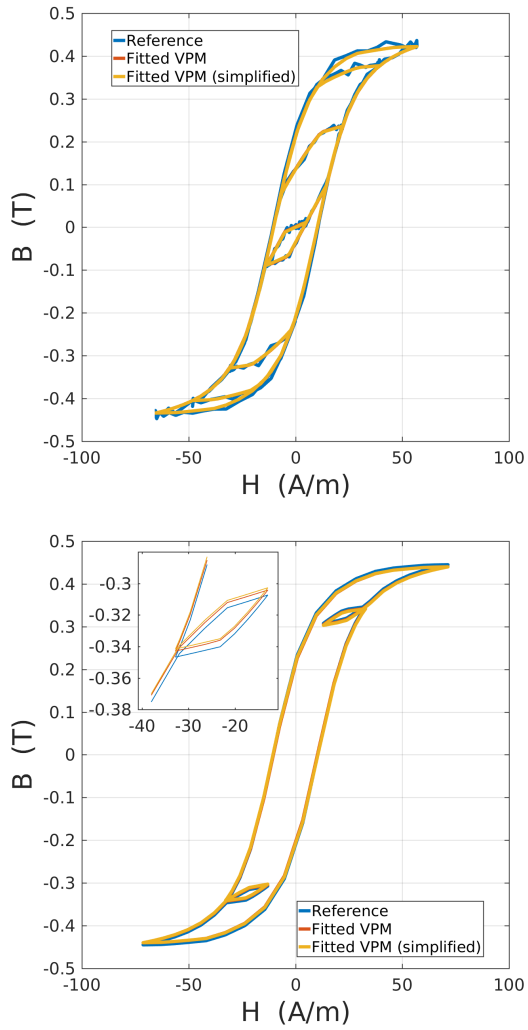

Figure 3: Left: simulated noisy measurement and fitted VPM by using formulation 13 with $N=10$. Right: simulation performed on a validation dataset with the ordinary (red) and simplified (yellow) model; in the inset is shown a zoom of a minor asymmetrical loop.

Table 1: Values of $\chi^{k}(\mathrm{~A} / \mathrm{m})$ obtained from simulations $(N=10)$

\begin{tabular}{crr}
\hline$k$ & eq. $[13)$ & eq. $[13]$, simplified \\
\hline 1 & 0 & 0 \\
2 & $4.681 \pm 0.729$ & 5.064 \\
3 & $5.064 \pm 0.484$ & 5.064 \\
4 & $5.409 \pm 0.938$ & 5.064 \\
5 & $13.427 \pm 2.148$ & 14.938 \\
6 & $14.496 \pm 0.695$ & 14.938 \\
7 & $14.745 \pm 0.458$ & 14.938 \\
8 & $14.938 \pm 0.530$ & 14.938 \\
9 & $15.152 \pm 0.658$ & 14.938 \\
10 & $15.454 \pm 0.950$ & 15.454 \\
\hline Number of cells: & 10 & 4 \\
\hline
\end{tabular}



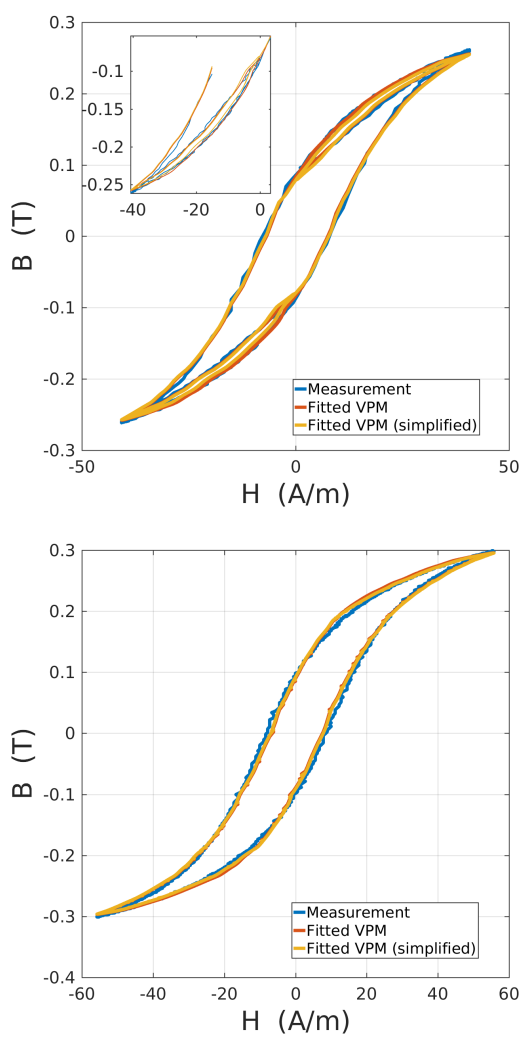

Figure 4: Left: measurement used to fit VPMs with $N=10$. Right: validation with a symetric minor loop. Red and yellow curves are the ouput generated respectively by the ordinary (red) and simplified (yellow) VPM.

Table 2: Values of $\chi^{k}(\mathrm{~A} / \mathrm{m})$ obtained for a $M n Z n$ N30 ferrite $(N=10)$

\begin{tabular}{crr}
\hline$k$ & eq. $\sqrt{13})$ & eq. $\sqrt{13}$, simplified \\
\hline 1 & 0 & 0 \\
2 & 0.0785 & 0 \\
3 & 0.1506 & 0.2171 \\
4 & 0.2171 & 0.2171 \\
5 & 0.2789 & 0.2171 \\
6 & 3.0845 & 3.0845 \\
7 & 5.8032 & 3.0845 \\
8 & 18.3351 & 22.4283 \\
9 & 21.4755 & 22.4283 \\
10 & 22.4283 & 22.4283 \\
\hline Number of cells: & 10 & 4 \\
\hline
\end{tabular}



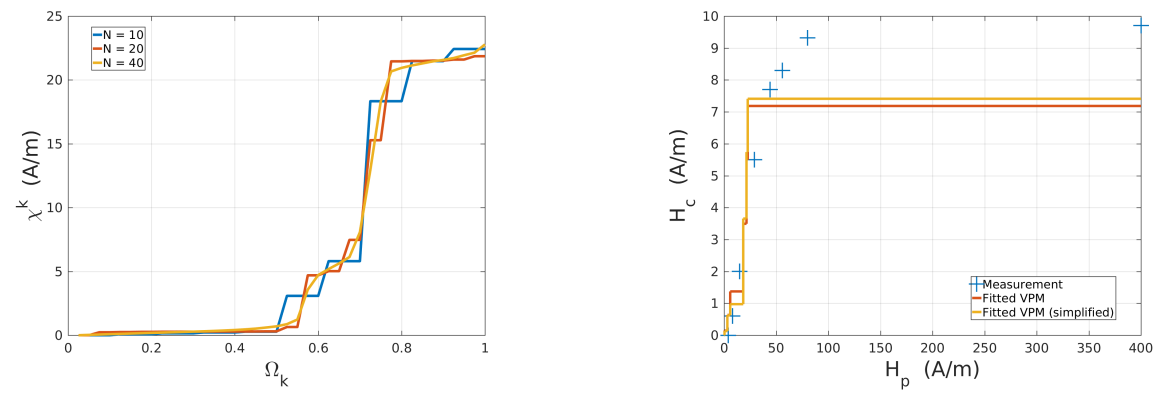

Figure 5: Left: estimated values of $\left(\chi^{k}\right)$ for VPMs with $N=10,20$ and 40 cells. Right: curve $H_{c}\left(H_{p}\right)$ computed from VPMs by using (10) (lines) and experimental measurements $(+)$.

simplification algorithm is very effective in reducing the number of cells of the VPM, yet ensuring a good agreement with data. Computations performed with higher values of $N$ provided quite similar results (figure 5 left).

We compared our approach with the one proposed in [8]. We found that the identified VPMs are compatible with the curve $H_{c}\left(H_{p}\right)$ for low values of $H_{p}$ (namely $H_{p}<60 \mathrm{~A} / \mathrm{m}$ ), whereas discrepancy is observed for higher values (figure 5 right).

We tried to identify the VPM by using a major cycle only, but we obtained a very poor result (not shown). We believe that this is due to the fact that cells with lowest values of $\chi^{k}$, which come to play with small loops, are the hardest to identify. When using large loops for identification, these cells come to play only when the field is reversed - that is for high values of the flux density, where the sensibility of measurements is low. Therefore, it can be argued that it is necessary to include small loops in the dataset used for identification.

\subsection{Various tests of the VPM with $h(t)$ and $T$}

We tested the VPM with several kind of signals $h$ and temperatures $T$. The set of parameters at room temperature are those mentioned in table 2 , with formulation (13) simplified and the anhysteretic magnetization functions $M_{a n}(h, T)$ and $\chi(T)$ that are used are those plotted in Fig 2 Figure6(left) shows measured and simulated major and minor symmetric loops at room temperature showing that the VPM is able to reproduce symmetric loops at room temperature with different levels of applied field. Figure 6 (right) shows that the VPM can simulate major loops at different temperatures thanks to the method mentioned in section 2 .

As the VPM is normally able to predict loops with any kind of $h(t)$ signals, we tested it with the applied field defined by 15 :

$$
h(t)=H_{\max }(\sin (\omega t)+\sin (3 \omega t))
$$

Simulated and measured loops are compared on figure 7 for two levels of $H_{\max }$. Simulated and measured results are in very good agreement showing that the set of identified parameters combined to the method defined in section 2 is 

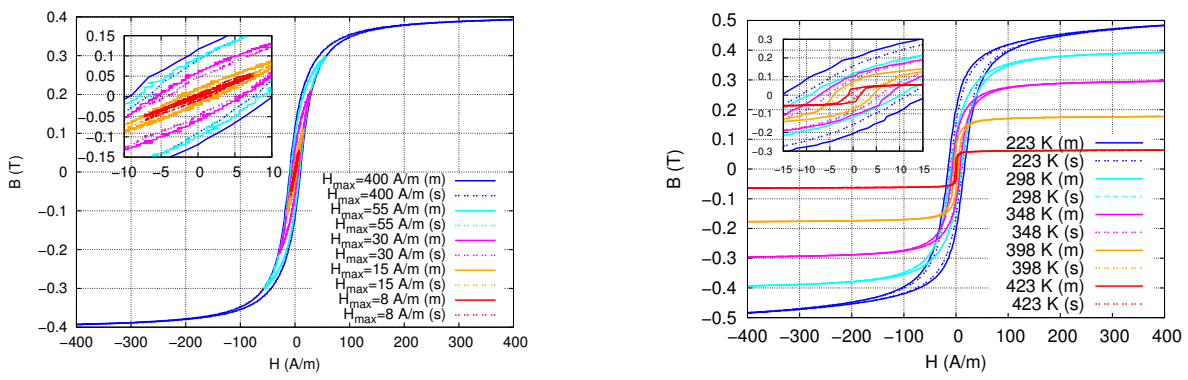

Figure 6: Left : major and minor symmetric loops at room temperature. Right : Major loops for different temperatures. Full lines : measured values ; dotted lines : simulated values

well adapted to predict loops with any kind of applied field waveform and any temperature within the range of measured $M_{a n}(T), H_{c}(T)$.
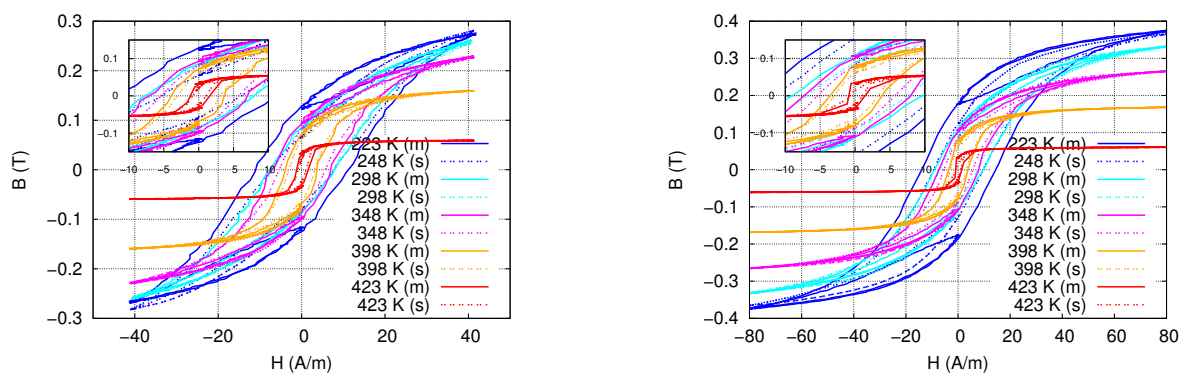

Figure 7: Left : loops with third harmonic $\left(H_{\max }=40 \mathrm{~A} / \mathrm{m}\right)$ at different temperatures. Right : loops with third harmonic $\left(H_{\max }=80 \mathrm{~A} / \mathrm{m}\right)$ at different temperatures. Full lines : measured values ; dotted lines : simulated values

Last but not least, we wanted to know if the VPM combined to its dependent extension was able to retrieve the Hopkinson effect 20. Loops are simulated at different temperatures for two applied field levels ( $h$ imposed). The tips of each loop is used to determine the per unit magnetic permeability $\mu_{r}$. Figure 8 (left) show $\mu_{r}$ variations with the temperature $T$ for two levels of applied field $\left(H_{\max }=8 \mathrm{~A} / \mathrm{m}\right.$ and $\left.H_{\max }=40 \mathrm{~A} / \mathrm{m}\right)$. When a low field $\left(H_{\max }=8 \mathrm{~A} / \mathrm{m}\right)$ is applied to the material, a peak of magnetic permeability $\mu_{r}$ is observed when $T$ is increasing just before the Curie temperature, then it drops to $\mu_{r} \approx 1$, at $T_{c}$ (Curie temperature). Figure 8 right), shows the evolution of some simulated loops during the increase of $T$ for the level $H_{\max }=8 \mathrm{~A} / \mathrm{m}$. The loops first expand $(T=[298,393] \mathrm{K})$ when heating then shrink $(T=410 \mathrm{~K})$. Applying a stronger field $\left(H_{\max }=20 \mathrm{~A} / \mathrm{m}\right)$ reduces the peak level, but doesn't change the drop. The global physical behaviour of the Hopkinson effect is qualitatively retrieved. Unfortunately measurements couldn't have been done to compare to the simulations. 

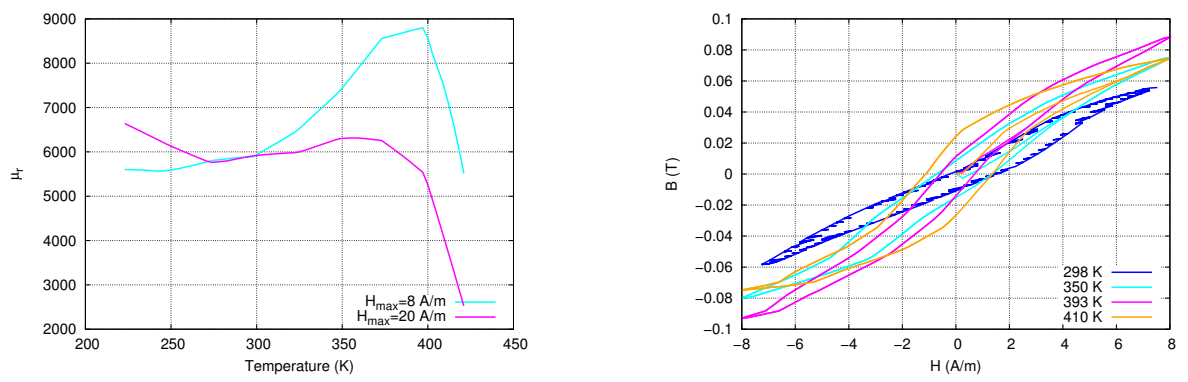

Figure 8: Left : relative permeability $\mu_{r}$ vs $T$. Right : minor loops at different temperatures showing the Hopkinson effect.

\section{Discussion}

The main purpose of this work is to describe how Vector Play Model (VPM) can effectively take into account the effect of temperature. In particular, it has been shown that VPM identified at room temperature can be used to model the behaviour of magnetic materials at any temperature, provided that few additional measurements have been performed in the range of temperature.

To this aim, we developed formulation (13) to identify the parameters of the VPM. Conversely to existing works $8,10,13$, this formulation has as unknowns only the parameters $\left(\chi^{k}\right)$, whereas $\omega_{k} \equiv 1 / N$ is fixed a priori.

As for the measurements required to identify VPMs, formulation 13 requires no particular signal: the only requirement is that datasets have to be "rich enough" for activating all cells in such a way that they can be discriminated. For instance, symmetric minor cycles will suit for this purpose, whereas the major cycle alone will not - the reason being that cells corresponding to small values of $\chi^{k}$ are activated for high values of the field, where the sensitivity is the poorest: so they can be hardly identified.

We compared our approach with the approach proposed in $[8$. We found that, according to our measurements, formulation 13 provides more accurate result, which is compatible with the $H_{c}\left(H_{p}\right)$ curve for low values of $H_{p}$. However, it has to be said that in our work VPMs have been identified by using a cycle, the peak excitation field of which is $45 \mathrm{~A} / \mathrm{m}$ : therefore it could be reasonable that some discrepancy may appear for higher values of $H_{p}$. This point merits to be more deeply investigated.

It has to be remarked that this formulation is very stable with respect of the number of cells $N$. The approach followed in this work is therefore to identify VPM with a high number of cells, and then simplify it by using the proposed (or others) algorithm, which has demonstrated to be very effective in reducing the complexity of the model. Moreover, this algorithm can be further improved: for instance, in the exemple discussed above it is clear that the last two cells could be packed together to obtain a 3-cells VPM:

$$
(0.1,0)-(0.3,5.064)-\underbrace{(0.5,14.938)-(0.1,15.454)}_{(0.6,15.024)}
$$

By the way, this approach does not require the a priori knowledge of the number of cells (which is needed with formulation (8)), or - said differently - it is possible 
to devise algorithms which determine the minimum number of cells which is required to achieve an imposed accuracy.

It is shown (figures 6 and 7 ) that the experimentally measured loops were very well retrieved by the VPM. This fact suggests that (at least for this material) the assumptions made at section 2 regarding the thermal behaviour are correct. It also points out that the $\chi^{k}$ identification protocol is a key element in order to have a good accuracy in a large range.

Concerning the Hopkinson effect, the VPM behaves qualitatively well due, we think to the following reasons. For low field levels, at low temperature only the smallest $\chi^{k}$ are activated (moving). When $T$ increase, all $\chi^{k}$ decrease and new cells can then be activated so the relative permeability $\mu_{r}$ increase until the material saturates. The peak of $\mu_{r}$ can then be seen as new cells that are activated in the material as $T$ increase. When the material is saturated, $\mu_{r}$ decrease because the saturation magnetization decrease very rapidly as $T$ is close to the Curie temperature (this is due to the dependence of $M_{a n}\left(h_{r e}, T\right)$ on $T$ ).

For higher field levels, all "small" $\chi^{k}$ cells are activated even at low temperature. The small increase of $\mu_{r}$ is, in this case, due to the small reduction of the large $\chi^{k}$ cells which tend to make the cells a bit more sensitive to the field. But no new cells are activated as $T$ increase. The decrease of $\mu_{r}$ is also due to the saturation, but arrives at lower temperature because the field level is higher.

\section{Conclusion and perspectives}

In this work we propose modifications to existing VPM so as to take into account the effect of temperature, as well as a new formulation for identifying VPM from experiments. Conversely to existing approaches [8, 21, our approach does not require a specific kind of measurement. Proposed formulation has been shown to be stable, and by using a simplification algorithm can provide accurate VPMs with a low number of cells.

Some discrepancy appeared between our results and the approach proposed by [8, which require some more work to be elucidated. The VPM associated with a 2D Lookuptable for $M_{a n}$ and a rescaling of the $\chi^{k}$ thanks to the macroscopic coercive field $H_{c}$ is now able to predict any loop for any kind of signals $h(t)$ for any temperature within the range. VPM has also shown its ability to retrieve qualitatively the Hopkinson effect.

Up to now, the proposed approach has been tested successfully with a single material: future works will be devoted to more extensive experimentation with different materials, so as to assess (or not) the effectiveness of our model.

Possible other extensions of the VPM model, following the same approach, may be envisaged. For example, simulate the influence of a mechanical stress on the magnetic properties by modifying $M_{a n}$ and $\chi^{k}$ following the same method and assumptions than here (lookup tables and rescaling).

Another possible extension would be to simulate dynamic hysteresis. Two separate ways can be imagined. The first one, would be to decompose the dynamic applied field in several fields such as Bertotti's theory 22 and to use the VPM to compute the static part of the field $H_{s}$. To do that, the VPM has to be inversed i.e calculate $H_{s}(B)$. Another way, would be to attribute a mass to the different cells in order to give inertia to the cells. 


\section{References}

[1] D. Jiles and D. Atherton, "Ferromagnetic hysteresis," IEEE Transactions on Magnetics, vol. 19, no. 5, pp. 2183-2185, 1983.

[2] F. Preisach, "Über die magnetische nachwirkung," Zeitschrift für Physik, vol. 94, no. 1, pp. 277-302, 1935.

[3] A. Raghunathan, Y. Melikhov, J. E. Snyder, and D. Jiles, "Theoretical model of dependence of hysteresis based on mean field theory," IEEE Transactions on Magnetics, vol. 46, no. 06, p. 1507 -1510, 2010.

[4] O. Messal, F. Sixdenier, L. Morel, and N. Burais, "Temperature dependent extension of the jiles-atherton model: Study of the variation of microstructural hysteresis parameters," IEEE Transactions on Magnetics, vol. 48, no. 10, pp. 2567-2572, 2012.

[5] A. Stancu and L. Spinu, "Temperature and time-dependent preisach model for a stoner-wohlfarth particle system," IEEE Transactions on Magnetics, vol. 34, pp. 3867-3875, Nov 1998.

[6] P. D. Mitchler, E. D. Dahlberg, E. E. Wesseling, and R. M. Roshko, "Henkel plots in a temperature and time dependent preisach model," IEEE Transactions on Magnetics, vol. 32, pp. 3185-3194, Jul 1996.

[7] A. Bergqvist, "Magnetic vector hysteresis model with dry friction-like pinning," Physica B: Condensed Matter, vol. 233, no. 4, pp. 342-347, 1997.

[8] F. Henrotte, A. Nicolet, and K. Hameyer, "An energy-based vector hysteresis model for ferromagnetic materials," COMPEL, vol. 25, no. 1, pp. $71-80$, 2006.

[9] V. François-Lavet, F. Henrotte, L. Stainier, L. Noels, and C. Geuzaine, "Vectorial incremental nonconservative consistent hysteresis model," in Proceedings of the 5th International Conference on Advanded COmputational Methods in Engineering (ACOMEN2011), (Liège, Belgium), ACOMEN2011, November 2011.

[10] V. François-Lavet, F. Henrotte, L. Stainier, L. Noels, and C. Geuzaine, "An energy-based variational model of ferromagnetic hysteresis for finite element computations," Journal of Computational and Applied Mathematics, vol. 246, pp. 243-250, 2013.

[11] A. Bergqvist, D. Lin, and P. Zhou, "Temperature-dependent vector hysteresis model for permanent magnets," IEEE Transactions on Magnetics, vol. 50, no. 2, p. 7008404, 2014.

[12] D. Lin, P. Zhou, and A. Bergqvist, "Improved vector play model and parameter identification for magnetic hysteresis materials," IEEE Transactions on Magnetics, vol. 50, no. 2, p. 7008704, 2014.

[13] F. Sixdenier, O. Messal, A. Hilal, C. Martin, M.-A. Raulet, and R. Scorretti, "Temperature-dependent extension of a static hysteresis model," IEEE Transactions on Magnetics, vol. 52, no. 03, p. , 2016. 
[14] P. Weiss, "La constante du champ moléculaire. equation d'état magnétique et calorimétrie," Journal de Physique, vol. 1, no. 5, pp. 163-175, 1930.

[15] R. Gersdorf, "Ferromagnetic properties of $\mathrm{Fe}$ and $\mathrm{Ni}$ in relation to their band structure.," J. Phys. Radium, vol. 23, no. 10, pp. 726-729, 1962.

[16] F. Fausto, Characterization and Measurement of Magnetic Materials. Academic Press, 2005.

[17] A. Wirgin, "The inverse crime," arXiv preprint math-ph/0401050, 2004.

[18] R. H. Byrd, M. E. Hribar, and J. Nocedal, "An interior point algorithm for large-scale nonlinear programming," SIAM Journal on Optimization, vol. 9, no. 4, pp. 877-900, 1999.

[19] R. C. Aster, B. Borchers, and C. H. Thurber, Parameter estimation and inverse problems, vol. 90. Academic Press, 2011.

[20] J. Hopkinson, "Magnetic and other physical properties of iron at a high temperature.," Philosophical Transactions of the Royal Society, vol. 180, no. 1, pp. 443-465, 1889.

[21] J. H. Krah and A. Bergqvist, "Numerical optimization of a hysteresis model," Physica B: Condensed Matter, vol. 343, no. 1, pp. 35-38, 2004.

[22] G. Bertotti, "General properties of power losses in soft ferromagnetic materials," IEEE Transactions on Magnetics, vol. 24, no. 1, pp. 621-630, 1988. 